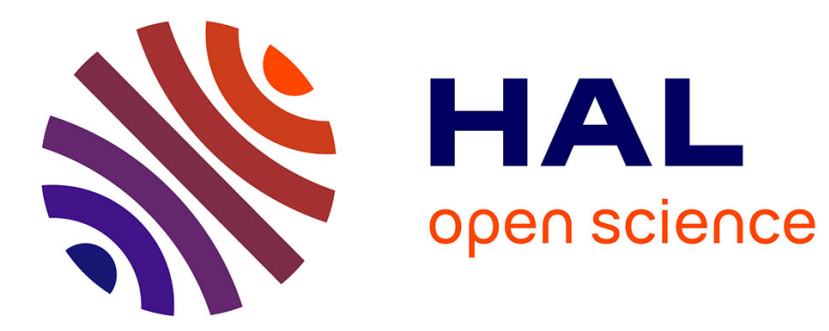

\title{
Couches épitaxiales d'arséniure de gallium obtenues par la technique de transport en phase vapeur
}

\author{
T.T. Anh Nhuyen, J. Vuillod
}

\section{To cite this version:}

T.T. Anh Nhuyen, J. Vuillod. Couches épitaxiales d'arséniure de gallium obtenues par la technique de transport en phase vapeur. Revue de Physique Appliquée, 1966, 1 (3), pp.161-163. 10.1051/rphysap:0196600103016100 . jpa-00242708

\section{HAL Id: jpa-00242708 https://hal.science/jpa-00242708}

Submitted on 1 Jan 1966

HAL is a multi-disciplinary open access archive for the deposit and dissemination of scientific research documents, whether they are published or not. The documents may come from teaching and research institutions in France or abroad, or from public or private research centers.
L'archive ouverte pluridisciplinaire HAL, est destinée au dépôt et à la diffusion de documents scientifiques de niveau recherche, publiés ou non, émanant des établissements d'enseignement et de recherche français ou étrangers, des laboratoires publics ou privés. 


\title{
COUCHES ÉPITAXIALES D'ARSÉNIURE DE GALLIUM OBTENUES PAR LA TECHNIQUE DE TRANSPORT EN PHASE VAPEUR
}

\author{
Par T. T. Anr NGUYEN et J. VUILLOD, \\ Laboratoire d'Électrostatique et de Physique du Métal, \\ Service Lames Minces, B. P. 319, Grenoble.
}

\begin{abstract}
Résumé. - On présente une étude de l'épitaxie de l'arséniure de gallium sur des supports de germanium réalisée par la technique de transport en phase vapeur. Cette étude comprend notamment des analyses cristallographiques faites par microscopie optique, par diffraction des rayons $X$ et par diffraction électronique et des mesures électriques, principalement de résistivité et de mobilité.
\end{abstract}

Abstract. - We have studied the epitaxy of gallium arsenide deposited by the vapor phase transport technique, on germanium. We treat in particular crystallographic analyses effected by optical microscopy, X-ray diffraction and electron diffraction, as well as electrical measurements of mobility and resistivity.

Introduction. - L'épitaxie de l'arséniure de gallium sur des supports de germanium est réalisée par la technique de transport en phase vapeur, dans des montages à tubes ouverts. Les gaz réactifs utilisés sont le $\mathrm{HCl}$ anhydre, la vapeur d'eau et le trichlorure d'arsenic.

Les valeurs optimales des paramètres permettant l'obtention d'une bonne épitaxie reproductible sont déterminées pour chaque montage. L'étude cristallographique des dépôts est faite par microscopie optique, par diffraction des rayons $\mathrm{X}$ et par diffraction électronique. Les propriétés électriques des couches sont évaluées, principalement par les mesures de résistivité et de mobilité de Hall.

L'arséniure de gallium est aussi déposé épitaxia. lement sur des supports d'arséniure de gallium. Les résultats préliminaires de cette étude sont cités.

Pour la synthèse des couches épitaxiales d'arséniure de gallium, la technique de transport par réactions chimiques en ph.sse vapeur est couramment utilisée. L'arsenic est vaporisé thermiquement. Le gallium, très peu volatil, est attaqué par un gaz réactif et transformé en composés plus volatils. Les agents de transfert les plus utilisés sont le gaz chlorhydrique [1], le trichlorure de allium [2], le trichlorure d'arsenic [3], et la vapeur d'eau [4].

Le mécanisme de transport, communément admis, se fait suivant les réactions d'équilibre suivantes :

$$
\begin{aligned}
& \mathrm{Ga}(1)+x \mathrm{HCl}(\mathrm{v}) \rightleftarrows \mathrm{GaCl}_{x}(\mathrm{v})+\frac{x}{2} \mathrm{H}_{2}(\mathrm{v}) \\
& 2 \mathrm{Ga}(1)+\mathrm{H}_{2} \mathrm{O}(\mathrm{v}) \rightleftarrows \mathrm{Ga}_{2} \mathrm{O}(\mathrm{v})+\mathrm{H}_{2}(\mathrm{v}) .
\end{aligned}
$$

On démontre thermodynamiquement qu'une augmentation de la température déplace ces réactions vers la droite et qu'une décroissance de la température favorise les réactions inverses. Le gallium, placé dans la zone la plus chaude du tube de réaction est transporté sous forme de chlorures ou d'oxyde de gallium volatils et déposé ensuite par le mécanisme de réactions inverses dans une zone de température plus basse. Dans cette partie, les atomes de gallium se combinent avec la vapeur d'arsenic présente pour former du GaAs.

Résultats expérimentaux. - Nous avons expérimenté quatre montages à tubes ouverts. Les deux premiers, l'un à source de gallium et d'arsenic séparées, l'autre à source unique de GaAs massif, utilisent comme agent de transport du $\mathrm{HCl}$ anhydre dilué dans un courant d'hydrogène. Le troisième, à sources séparées, emploie la vapeur d'eau comme gaz réactif. Dans le quatrième, dont la source est constituée soit de gallium métallique, soit de GaAs, le gaz chlorhydrique est formé à l'intérieur même du tube de réaction par décomposition thermique de la vapeur de trichlorure d'arsenic en présence ${ }_{\mathrm{s}}^{\mathrm{m}} \mathrm{d}$ 'hydrogène.

Notre étude porte principalement sur les dépôts effectués sur des supports de germanium, mais nous avons commencé une série d'expériences avec des supports de GaAs (111), de type $N$, non dopés. Les plaquettes de germanium utilisées sont d'orientation (111), (110) et (100), de deux types $N$ et $P$, et de résistivités comprises entre $0,006 \Omega . \mathrm{cm}$ et $30 \Omega$.cm.

$1^{0}$ Épitaxie. - Les paramètres suivants : températures des sources et du substrat, gradient de température, vitesse d'écoulement du gaz dans le tube de réaction et teneur en agent réactif du mélange gazeux sont trouvés critiques. Nous avons fait une étude systématique de ces paramètres, qui sont liés les uns aux autres, pour déterminer les conditions optimales d'épitaxie.

Lorsque le gradient de température dépasse $150^{\circ} \mathrm{C}$ 
environ, les dépôts sont non uniformes, polycristallins et recouverts de whiskers. Alors, même avec des supports portés aux températures relativement élevées, $800^{\circ} \mathrm{C}$ par exemple, nous n'avons obtenu aucune couche monocristalline. Pour des gradients compris entre $80^{\circ} \mathrm{C}$ et $150^{\circ} \mathrm{C}$, et pour les températures $T_{\mathrm{s}}$ des supports de germanium supérieures à $680^{\circ} \mathrm{C}$ environ, les dépôts sont monocristallins. Quand on abaisse $T_{\mathrm{s}}$ ces dépôts deviennent d'abord mâclés puis ensuite polycristallins. La planéité de la surface augmente avec $T_{\mathrm{s}}$, mais dans le cas du germanium et des montages utilisant le gaz chlorhydrique comme agent de transport, nous sommes limités par une valeur supérieure, qui est de $730^{\circ} \mathrm{C}$, température d'eutectique du système $\mathrm{Ge}-\mathrm{As}$ au-delà de laquelle la surface est souvent tachetée en de nombreux endroits qui semblent être des points de recristalisation d'une phase solide-liquide.

L'épitaxie est obtenue de manière reproductible dans les conditions suivantes : sources $\mathrm{Ga}$ ou $\mathrm{GaAs}$ portées à $800-850{ }^{\circ} \mathrm{C}$, arsenic à $400-450^{\circ} \mathrm{C}$, température des supports de germanium $680-720$ oC, débit d'hydrogène de dilution $10 \mathrm{l} / \mathrm{h}$. La vitesse moyenne de croissance sur des supports (111) est de 25 microns à l'heure quand le débit de $\mathrm{HCl}$ est de $0,36 \mathrm{l} / \mathrm{h}$ et de 10 microns à l'heure lorsqu'un courant d'hydrogène de $3,6 \mathrm{l} / \mathrm{h}$ barbote dans du $\mathrm{AsCl}_{3}$ maintenu à $24^{\circ} \mathrm{C}$.

Les conditions d'obtention d'épitaxie avec le transport par la vapeur d'eau sont différentes des précédentes. Le gallium doit être plus fortement chauffé et, alors que dans les autres montages le dépôl se forme principalement dans le deuxième palier de température, il commence ici dans la partic supérieure du gradient. Des couches épitaxiées (111) de 15 microns d'épaisseur sont obtenues après une heure et demie de manipulations avec les valeurs suivantes des paramètres : source $\mathrm{Ga}$ à $870^{\circ} \mathrm{C}$, température au milieu du support $780^{\circ} \mathrm{C}$, hyúrogène de dilution $150 \mathrm{cc} / \mathrm{mn}$, hydrogène de barbotage $140 \mathrm{cc} / \mathrm{mn}$. La surface des dépôts est oxydée par endroits.

Les résistivités et la nature du dopant des supports ne semblent pas avoir une influence sur les caractéristiques cristallographiques des dépôts. Par contre, l'orientation joue un rôle prépondérant. Dans les mêmes conditions de dépôt, les films (100) présentent les surfaces les plus planes, viennent ensuite les films (110) et (111).

La vitesse de croissance dépend aussi de l'orientation des substrats. Par rapport aux faces (111), le dépôt se fait, en moyenne, deux fois plus rapidement sur les faces (100) et une fois et demie plus rapidement sur les faces $(110)$.

Les photographies 1, 2 et 3 donnent l'état caractéristique des surfaces des dépôts (111), (100) et (110) obtenus à $700^{\circ} \mathrm{C}$. L'étude en diffraction électronique (photo no 4) montre que ces couches sont monocristallines.

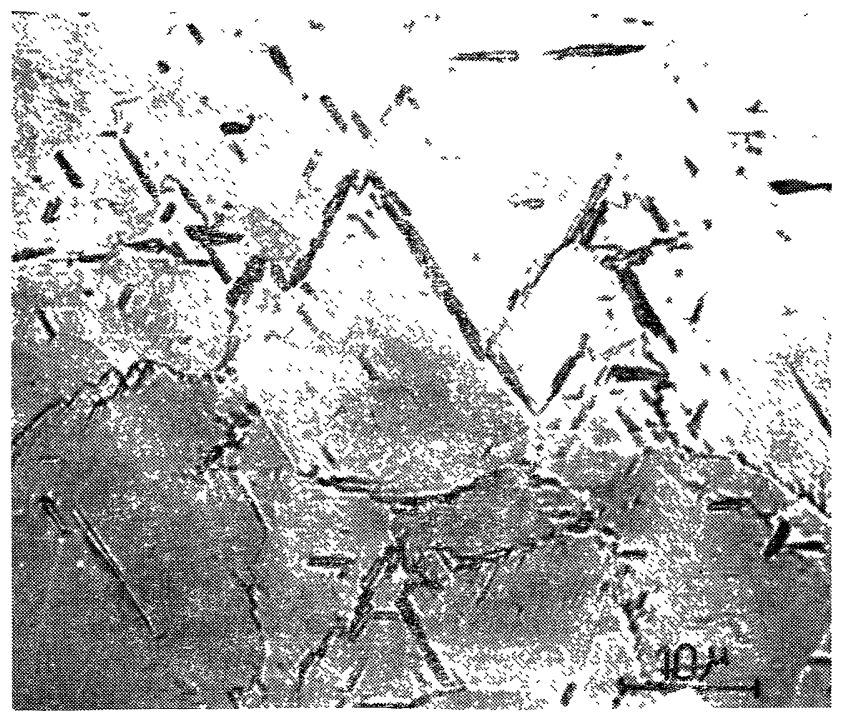

FIG. 1. - Ga $\backslash$ s (111)/Ge (111), $T_{\mathrm{s}}=700^{\circ} \mathrm{C}$.

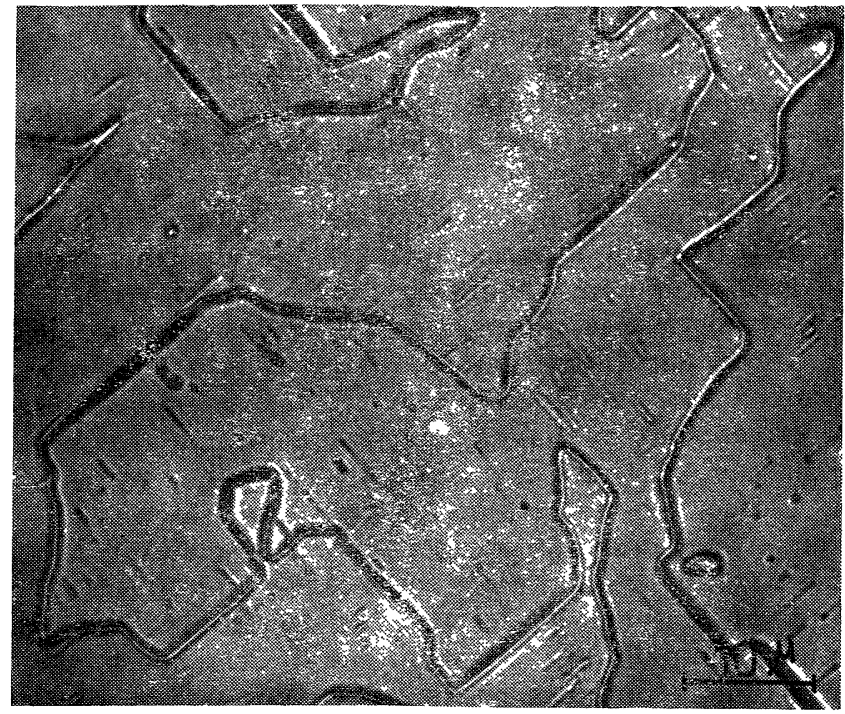

FIg. 2. - GaAs $(100) / \mathrm{Ge}(100), T_{\mathrm{s}}=700^{\circ} \mathrm{C}$.

Nous avons aussi déposé épitaxialement du GaAs sur des supports de GaAs ( $(\overline{1} \overline{1})$. Dans ce cas, les substrats doivent être portés à une température égale ou supérieure à $730^{\circ} \mathrm{C}$ pour que les couches soient monocristallines. Une telle couche est montrée par la photo $n^{0} 5$.

$2^{\circ}$ Caractéristiques électriques. - Des mesures non destructives de résistivité et de mobilité de Hall ont permis une caractérisation rapide des dépôts épitaxiaux d'arséniure de gallium. Ainsi avons-nous utilisé la méthode des quatre pointes pour mesurer les résistivités et une méthode de mesure utilisant l'effet Corbino pour déterminer la mobilité de Hall. D'autres mesures ont été entre- 


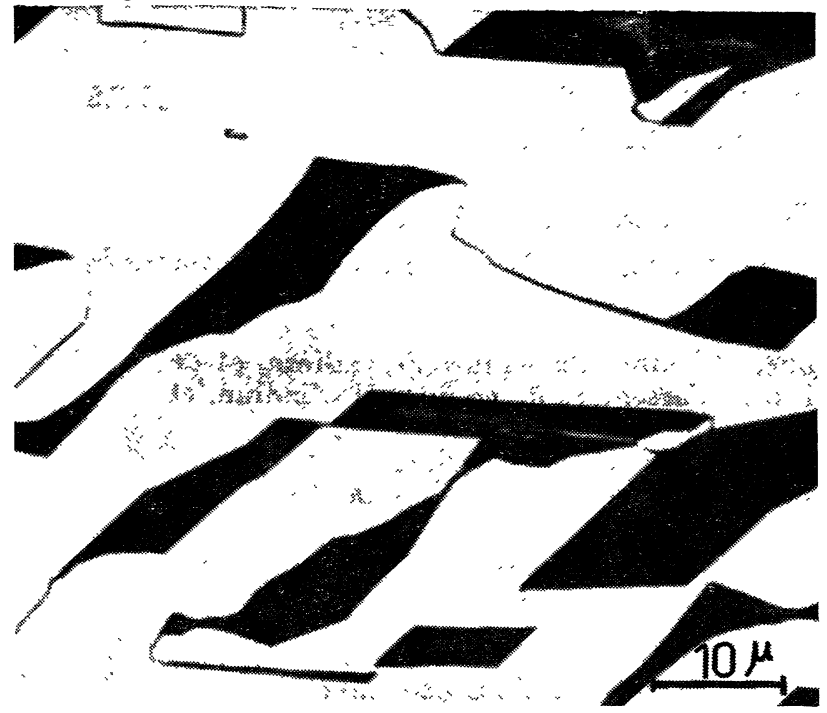

FIG. 3. $-\mathrm{GaAs}(110) / \mathrm{Ge}(110), T_{\mathrm{s}}=700^{\circ} \mathrm{C}$.

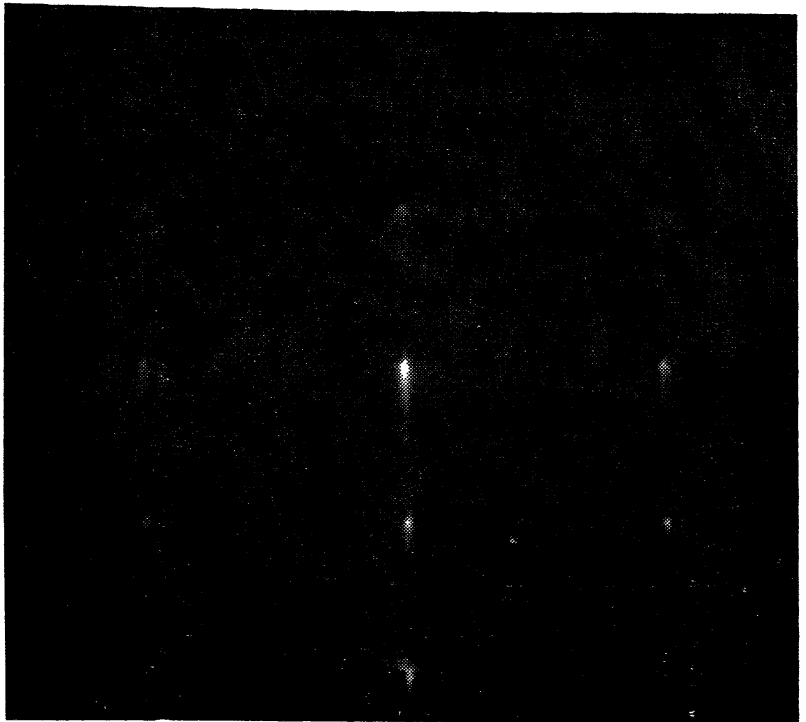

FIg. 4. - Diagramme de diffraction électronique d'une couche de GaAs (111) déposée sur Ge (111).

prises par les méthodes classiques nécessitant la réalisation de contacts ohmiques et ont donné des résultats comparables.

Toutes les couches obtenues sont de type $\mathrm{N}$.

La principale source d'impuretés provient du gaz réactif utilisé. En employant du gaz chlorhydrique Péchiney nous avons obtenu des mohilités de l'ordre

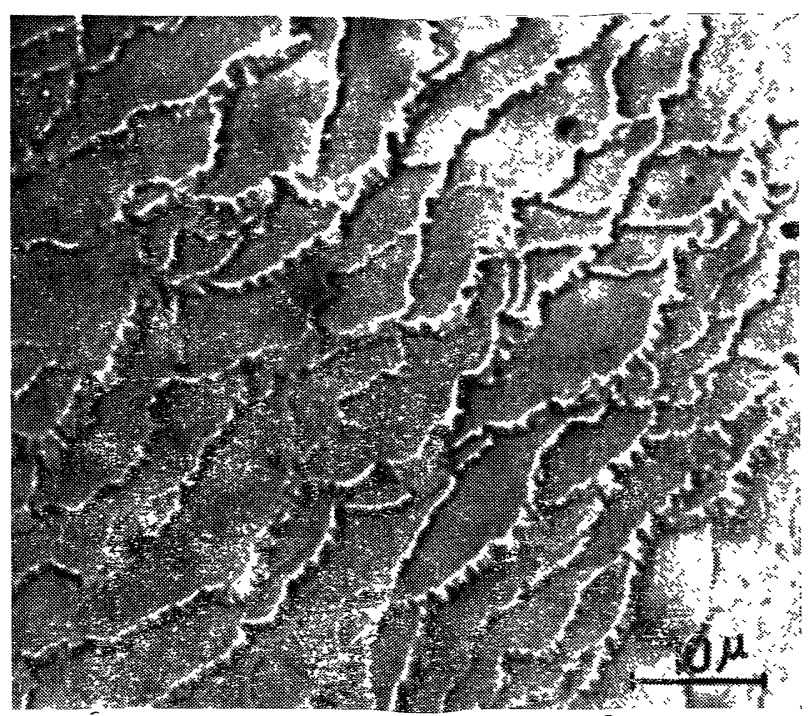

FIg. 5. - Dépôt de GaAs sur GaAs (111), $T_{\mathrm{s}}=733^{\circ} \mathrm{C}$

de la centaine de $\mathrm{cm}^{2} \mathbf{v}^{-1} \mathrm{~s}^{-1}$ et des résistivités de quelques millièmes d'ohm-cm avec le montage à sources séparées, et des valeurs deux fois plus grandes avec le montage à source unique. Ces valeurs ont pratiquement doublé lorsqu'on a utilisé du gaz HCl généré sur place à partir des acides chlorhydrique et sulfurique. L'utilisation de vapeur d'eau comme agent de transport donne des couches possédant de meilleures propriétés électroniques. Malheureusement, comme nous l'avons vu, ces dépôts sont oxydés par endroits.

Avec le montage au trichlorure d'arsenic, les mobilités dépassent le millier de $\mathrm{cm}^{2} \mathrm{v}^{-1} \mathrm{~s}^{-1}$ et les résistivités, plusieurs centièmes d'ohm-cm. Les meilleures valeurs ont été obtenues avec des couches déposées à $680-690^{\circ} \mathrm{C}$; elles diminuent en général Jorsque les supports sont portés à une température plus élevée.

Des hétérojonctions de GaAs $N$ sur supports de Ge $P$ sont en cours d'étude. Les résultats préliminaires obtenus montrent un net effet de redressement.

Des déterminations de profils d'impuretés dans le dépôt sont entreprises par des mesures de capacité en fonction de la polarisation inverse appliquée. Une diode à barrière de surface est réalisée en évaporant des points d'or sur le GaAs. Les premiers résultats obtenus indiquent une concentration d'impuretés $N_{\text {D }}-N_{\text {A }}$ de l'ordre de $10^{17} / \mathrm{cm}^{3}$ et semblent suggérer l'existence d'un phénomène de compensation dans ces couches.

\section{RÉFÉRENCES}

[1] Ing (S. W.) et Minden (H. T.), J. Electrochem. Soc., 1962, 109, 995.

[2] Goldsmith (N.) et Oshinky (W.), RCA Review, 1963, 24, 546.
[3] Finch (W. F.) et Menal (E. W.), J. Electrochem. Soc., 1964, 111, 814.

[4] Frosch (C. J.) et Thurmond (C. D.), Electrochem. Soc. Meeting, Boston, septembre 1962. 